\title{
Risk factors for symptomatic osteonecrosis in childhood ALL: A retrospective study of a Slovenian pediatric ALL population between 1970 and 2004
}

\author{
NATAŠA KARAS-KUŽELIČKI ${ }^{1}$, SIMONA MENCEJ-BEDRAČ ${ }^{1}$, JANEZ JAZBEC $^{2}$, \\ JANJA MARC $^{1}$ and IRENA MLINARIČ-RAŠČAN ${ }^{1}$
}

\author{
${ }^{1}$ Department of Clinical Biochemistry, Faculty of Pharmacy, University of Ljubljana; ${ }^{2}$ Unit of Oncology and Hematology, \\ University Medical Centre, University Children's Hospital, 1000 Ljubljana, Slovenia
}

Received July 27, 2015; Accepted April 4, 2016

DOI: $10.3892 /$ etm.2016.3391

\begin{abstract}
Treatment induced non-traumatic osteonecrosis (ON) has been reported increasingly in children treated for acute lymphoblastic leukemia (ALL). Several risk factors for ON have been identified in childhood cancer patients; however, their diagnostic and prognostic power is limited and the etiology of the disease remains unclear. Therefore, a continuous effort is focused on the identification of additional ON risk factors. We performed a retrospective study of 313 childhood ALL patients to test the association between the $\mathrm{ON}$ occurrence in children receiving ALL therapy and common polymorphisms in potential target genes: Thiopurine S-methyltransferase (TPMT; 460G>A, 719A>G), 5,10-methylenetetrahydrofolate reductase $(M T H F R ; 677 \mathrm{C}>\mathrm{T}, 1298 \mathrm{~A}>\mathrm{C})$, estrogen receptor alpha 1 (ESR1; XbaI) and collagen type I, $\alpha 1$ (COL1A1; Sp1). In the present cohort, higher age and more recently developed treatment protocols were independent risk factors for $\mathrm{ON}$. In children $>14.5$ years old, TPMT genotype modulated the risk of ON. Additionally, in children $<12.9$ years old ESRI genotypes were also implicated in the pathogenesis of $\mathrm{ON}$. Besides greater age and more recent treatment protocols,
\end{abstract}

Correspondence to: Dr Nataša Karas-Kuželički, Department of Clinical Biochemistry, Faculty of Pharmacy, University of Ljubljana, Aškerčeva 7, 1000 Ljubljana, Slovenia

E-mail: natasa.karas@ffa.uni-lj.si

Abbreviations: ACP-1, acid phosphatase 1; ALL, acute lymphoblastic leukemia; BFM, Berlin-Frankfurt-Münster; BMD, bone mineral density; COL1A1, collagen, type I, $\alpha 1$; ESR1, estrogen receptor alpha 1; GC, glucocorticoide; 6-MP, 6-mercaptopurine; MSC, mesenchimal stem cell; MTHFR, 5,10-methylenetetrahydrofolate reductase; MTX, methotrexate; ON, osteonecrosis; PAI-1, plasminogen activator inhibitor-1; POG, pediatric oncology group; TPMT, thiopurine S-methyltransferase; TYMS, tymidylate synthetase

Key words: acute lymphoblastic leukemia, collagen, type I, $\alpha 1$, estrogen receptor alpha 1, 5,10-methylenetetrahydrofolate reductase, osteonecrosis, thiopurine S-methyltransferase genetic factors (polymorphisms in ESRI and TPMT genes) were suggested to be implicated in the pathogenesis of $\mathrm{ON}$ and could be potentially used as genetic prognostic markers for ON.

\section{Introduction}

Osteonecrosis $(\mathrm{ON})$, also known as aseptic or avascular necrosis of the bone, is a disorder characterized by segmental death of one or more osseous sites (1). Although non-traumatic $\mathrm{ON}$ is rare in young people, it has been reported increasingly in children treated for acute lymphoblastic leukemia (ALL), as ALL survival rates continue to improve (1). The symptoms of $\mathrm{ON}$ are highly variable, ranging from mild discomfort to decreased mobility, severe pain and articular collapse (2). Although up to $25 \%$ of children treated for ALL exhibit radiographic evidence of osteopenia (3) and one-third of ALL patients develop symptomatic ON (1), the majority of patients are asymptomatic, some showing the spontaneous regression or even complete resolution of the disorder (1). Due to heterogenous diagnostic criteria, the reported incidence of $\mathrm{ON}$ varies widely in children with cancer; between 0.3 and $9 \%(2,4)$. The pathogenesis of $\mathrm{ON}$ is complex and includes the enhanced differentiation of mesenchymal stem cells (MSCs) into lipocytes at the expense of osteogenesis, as well as damage to the venous system, vascular stasis and ischemia (1). Several risk factors for $\mathrm{ON}$, such as older age, white race and glucocorticoid therapy (1), have been identified in infant cancer patients; however, their diagnostic power is limited and the etiology of the disease remains unclear. Therefore, there is a continuous effort to identify additional ON risk factors. In addition to glucocorticoids (GCs), other therapeutic agents such as methotrexate (MTX) and 6-mercaptopurine (6-MP) have been indicated in bone morbidity during ALL treatment (5-7). Recently, several polymorphisms have been identified as potential risk factors for $\mathrm{ON}$ during ALL treatment in genes involved in various processes, including: Bone metabolism, vitamin D receptor (8), collagen type II, $\alpha 1$ (1) and acid phosphatase 1 (ACP-1) (9); thrombosis, 5,10-methylenetetrahydrofolate reductase $(M T H F R)(9)$ and plasminogen activator inhibitor-1 (PAI-1) (10); and ALL pharmacogenetics, tymidylate synthetase (TYMS) (8). The present 
exploratory retrospective study was conducted to assess whether common polymorphisms in the target genes thiopurine S-methyltransferase (TPMT), MTHFR, estrogen receptor alpha 1 (ESRI) and collagen type I, $\alpha 1$ (COL1AI) are associated with $\mathrm{ON}$ in children receiving ALL therapy and could be used as prognostic genetic markers for ON. Target genes were selected to investigate all three aspects of ON risk: Thrombotic (MTHFR, ESR I); bone metabolism (ESRI, COLIAl and $M T H F R$ ); and pharmacogentic (TPMT, MTHFR). TPMT and MTHFR are key enzymes in the metabolism of 6-MP and MTX (11), respectively, and are crucial to the majority of ALL treatment protocols (12). They are administered in all phases of the therapy and are the primary drugs used in the maintenance phase, during which ON typically emerges (12). In addition, MTHFR variants $(9,13)$, as well as hyperestrogenemia (11), have been implicated in ON due to thrombophilia in adults. Finally, ESR 1 and COL1A1 were associated with bone mineral density (BMD) and other osteoporotic phenotypes in candidate gene studies (14), in addition to large-scale association $(15,16)$ and genome-wide studies $(17,18)$. Additionally, MTHFR has been suggested to be associated with osteoporotic phenotype in certain studies (17-19).

\section{Materials and methods}

Patients. Slovenian pediatric ALL patients diagnosed and treated at the University Children's Hospital, University Medical Centre (Ljubljana, Slovenia) between 1970 and 2004 were identified via the national oncology patients registry. Among the 405 registered patients with childhood ALL, 62 had inadequate medical records, 3 patients received non-Hodgkin's lymphoma Berlin-Frankfurt-Münster (NHL-BFM) treatment, 3 underwent bone marrow transplantation prior to maintenance therapy and 15 patients succumbed or relapsed prior to receiving maintenance therapy. A further 9 patients were later excluded from the study due to unsuccessful DNA extraction. The final study group consisted of 313 patients with childhood ALL. The study was conducted in accordance with the World Medical Association Declaration of Helsinki regarding ethical conduct of research involving human subjects. Ethical approval was obtained from the Medical Ethics Committee of Slovenia.

Different therapy protocols were applied in the study periods from 1970 to 2004. Pediatric oncology group (POG) protocols (20) were used from 1970 to 1983, and thereafter BFM protocols [BFM-83 (21), -86 (22), -90 (23), -95 (24) and intercontinental (IC) trial-BFM 2002 (25)]. Therapy data, which were systematically recorded by attending physicians, such as incidence of toxic effects, were obtained for 313 childhood ALL patients. From patients' medical records, 12 patients with symptomatic ON were identified, corresponding to grades 3 and 4 adverse events of NCI Common Toxicity Criteria (version 2.0) (26).

Genotyping. Genotyping was performed after the therapy data were extracted from patients' medical files and analyzed by researchers who were blinded to patients' medical data.

DNA was extracted from archival bone marrow smears of patients at the diagnosis of ALL using a semi-automated ABI PRISM $^{\mathrm{TM}} 6100$ Nucleic Acid PrepStation (Applied Biosystems; Thermo Fisher Scientific, Inc., Foster City, CA, USA).
TPMT ("3B: 460G>A, rs1800460 and *3C: 719A>G, rs1142345), MTHFR (677C $>$ T, rs1801133 and 1298A $>$ C, rs1801131), ESR1 (XbaI, rs9340799) and COL1A1 (Spl, rs1800012) polymorphisms were determined using TaqMan chemistry on ABI Prism ${ }^{\circledR} 7000$ Sequence Detection system (Applied Biosystems; Thermo Fisher Scientific, Inc.). TaqMan chemical reagents were purchased from Applied Biosystems). As $460 \mathrm{G}>\mathrm{A}$ and $719 \mathrm{~A}>\mathrm{G}$ polymorphisms in TPMT are in linkage disequilibrium and inherited together in cis, patients carrying both polymorphisms were considered to be heterozygotes for TPMT $^{*} 3 \mathrm{~A}$ allele. All low activity TPMT alleles were designated as TPMT*3 and wild-type as TPMT*1. We combined MTHFR $677 \mathrm{C}>\mathrm{T}$ and $1298 \mathrm{~A}>\mathrm{C}$ genotypes and established that $677 \mathrm{~T}$ rarely occurred in cis with $1298 \mathrm{C}$. In the group of 313 patients, only a single patient $(0.3 \%)$ exhibited the 677TT/1298AC genotype, which is in accordance with previous results (27). The 677CC/1298AA genotype was designated a wild type and all other as mutated genotypes.

Statistical analysis. To assess differences in age, gender, treatment protocol, TPMT, MTHFR, ESRI and COLIAl genotypes between $\mathrm{ON}$ and non-ON group the Fisher exact test was used.

For multivariate analysis, we applied the classification tree method to determine the interaction between multiple predictive variables and to evaluate the risk of $\mathrm{ON}$ associated with specific subgroups. ON was treated as the dependent variable, while all other variables (age, gender, treatment protocol and genotypes) were treated as independent variables. All variables were considered when constructing the classification tree. The classification and regression tree growing method was used and the minimum number of cases in parent and child node was set to 5 and 2, respectively. Due to the small number of ON cases the results of the classification tree were confirmed using Fisher exact test. $\mathrm{P}<0.05$ was considered to indicate a statistically significant difference. All statistical analyses were performed using the SPSS software, version 16.0 for Windows (SPSS, Inc., Chicago, IL, USA).

\section{Results}

Characteristics of the patients. The study group consisted of 313 childhood ALL patients, 12 (3.8\%) of whom were found to have had symptomatic ON (NCI grades 3 and 4). In the group of 313 childhood ALL patients, the mean age at ALL diagnosis was 5.9 \pm 4.2 years (range, 0.2-17.0 years). There were 148 (47.3\%) female and $165(52.7 \%)$ male patients. Furthermore, 94 (30.0\%), 37 (11.8\%), 55 (17.6\%), 59 (18.8\%), 55 (17.6\%) and $13(4.2 \%)$ of patients were treated with POG, BFM-83, -86, -90, -95 and IC-BFM 2002 protocols, respectively (Table I).

The genotyping for TPMT and MTHFR polymorphisms was successful for all 313 patients, while ESRI and COL1A1 genotypes were not determined for 23 and 52 patients, respectively, due insufficient extracted DNA from bone marrow smears. With respect to TPMT genotype, there were no homozygous mutated $(* 3 / 3)$ patients, $21(6.7 \%)$ patients were heterozygotes $\left({ }^{*} 1 /{ }^{*} 3\right)$ and the remainder were wild-type $\left({ }^{*} 1 /{ }^{*} 1\right)$. A total of $32(10.2 \%)$ patients were MTHFR wild type, while $281(89.8 \%)$ had at least one mutated allele in one of the two MTHFR locus. Among the 261 patients that were successfully genotyped for COL1A1, 201 (77.0\%) had GG, 51 (19.5\%) GT 
Table I. Analysis of independent risk factors for osteonecrosis in acute lymphoblastic leukemia patients with and without osteonecrosis.

\begin{tabular}{|c|c|c|c|}
\hline Parameter & Osteonecrosis $(\mathrm{N}=12)$ & No osteonecrosis $(\mathrm{N}=301)$ & $\mathrm{P}$ \\
\hline Age, years & $11.3 \pm 5.9$ & $5.6 \pm 4.0$ & 0.007 \\
\hline Gender, n (\%) & & & 0.774 \\
\hline Male & $7(58.3)$ & $158(52.5)$ & \\
\hline Female & $5(41.7)$ & $143(47.5)$ & \\
\hline Treatment protocol, n (\%) & & & 0.018 \\
\hline POG & $2(16.7)$ & $92(30.6)$ & \\
\hline BFM-83 & $0(0.0)$ & $37(12.3)$ & \\
\hline BFM-86 & $0(0.0)$ & $55(18.3)$ & \\
\hline BFM-90 & $5(41.7)$ & $54(17.9)$ & \\
\hline BFM-95 & $3(25.0)$ & $52(17.3)$ & \\
\hline IC-BFM 2002 & $2(16.7)$ & $11(3.7)$ & \\
\hline TPMT genotype, $\mathrm{n}(\%)$ & & & 0.188 \\
\hline${ }^{*} 1 /{ }^{*} 1$ & $10(83.3)$ & $282(93.7)$ & \\
\hline$* 1 / * 3$ & $2(16.7)$ & $19(6.3)$ & \\
\hline MTHFR genotype, n (\%) & & & 0.620 \\
\hline Wild type $^{\mathrm{a}}$ & $0(0.0)$ & $32(10.8)$ & \\
\hline Mutated $^{\mathrm{b}}$ & $12(100)$ & $269(89.4)$ & \\
\hline ESRl genotype, n (\%) & & & 0.465 \\
\hline GG & $4(33.3)$ & $53(19.1)$ & \\
\hline GA & $4(33.3)$ & $121(43.5)$ & \\
\hline AA & $4(33.3)$ & $104(37.4)$ & \\
\hline COL1A1 genotype, n (\%) & & & 0.797 \\
\hline GG & $10(90.9)$ & $191(76.4)$ & \\
\hline GT & $1(9.1)$ & $50(20.0)$ & \\
\hline $\mathrm{TT}$ & $0(0.0)$ & $9(3.6)$ & \\
\hline
\end{tabular}

${ }^{\mathrm{a}} 677 \mathrm{CC} / 1298 \mathrm{AA} .{ }^{\mathrm{b}} \mathrm{All}$ other genotype combinations with at least one mutated allele. Independent samples t-test were used for the age and Fisher's exact test was used for all the other parameters. POG, pediatric oncology group; BFM, Berlin-Frankfurt-Münster; IC, intercontinental; TPMT, thiopurine S-methyltransferase; MTHFR, 5,10-methylenetetrahydrofolate reductase; ESR1, estrogen receptor alpha 1; COL1A1, collagen, type I, $\alpha 1$.

and 9 (3.4\%) TT genotype. The distribution of ESR1 genotypes in the 290 successfully genotyped patients was as follows: GG, 57 (19.7\%); AG, 125 (43.1\%); and AA, 108 (37.2\%). All examined polymorphisms were in Hardy-Weinberg equilibrium.

Independent risk factors for $O N$. To identify independent risk factors for $\mathrm{ON}$, we compared all variables (age, gender, treatment protocol and studied genotypes) between $\mathrm{ON}$ patients and non-ON patients. Higher age at diagnosis and more recent treatment protocols were identified as independent risk factors for ON (Table I and Fig. 1).

Combined risk factors for $O N$. To investigate the interaction between multiple risk factors, we employed classification tree analysis (Fig. 2). The terminal nodes of the classification tree with the highest incidence of $\mathrm{ON}$ represented the patient subgroups with the highest risk for $\mathrm{ON}$.

The subgroup with highest (100\%) risk for ON consisted of ALL patients $>14.5$ years old with the mutated TPMT genotype. The ON incidence in this subgroup was compared with its incidence in the remainder of the observed population by means of Fisher exact test, which showed a statistically significant difference $(\mathrm{P}=0.001)$ (Fig. 3). The group with the second highest risk (50\%) for ON consisted of patients 2.4-12.9 years old, who had GG ESRl genotype and were treated with protocols BFM-95 and IC-BFM $2002(\mathrm{P}=0.075)$. Patients aged 2.2-2.4 years with ESR1 GG and AG genotypes represented the third risk group with $\mathrm{ON}$ incidence of $40 \%(\mathrm{P}=0.013)$. The incidence of $\mathrm{ON}$ in all terminal nodes and its comparison to the remainder of the observed population is shown in Fig. 3. Notably, a combination of age 2.4-12.9 years and ESRI AA and AG genotypes had a protective effect, as none of the 185 patients in this subgroup developed symptomatic ON $(\mathrm{P}<0.001)$.

\section{Discussion}

In the studied population of childhood ALL patients, the incidence of ON between 1970 and 2004 was 3.8\%. Greater age and later treatment protocols were independent risk factors for 

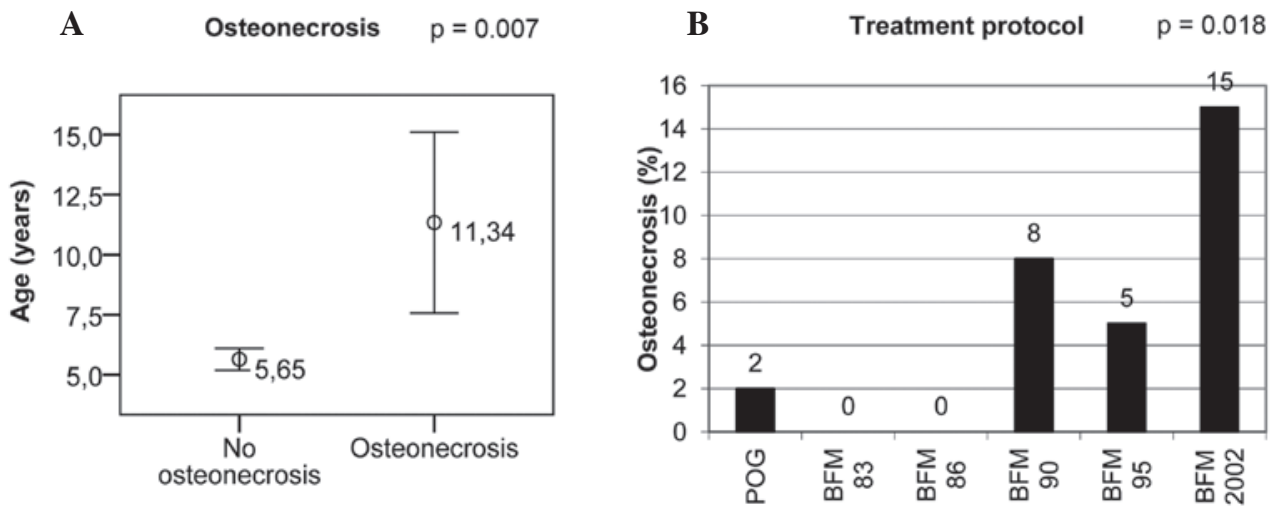

Figure 1. Independent risk factors for osteonecrosis. (A) Age and (B) treatment protocol were independent risk factors for osteonecrosis in childhood acute lymphoblastic leukemia patients; error bars represent $95 \%$ confidence interval of the mean. Independent samples t-test were used for the age and Fisher's exact test was used for the treatment protocols. POG, pediatric oncology group; BFM, Berlin-Frankfurt-Münster.

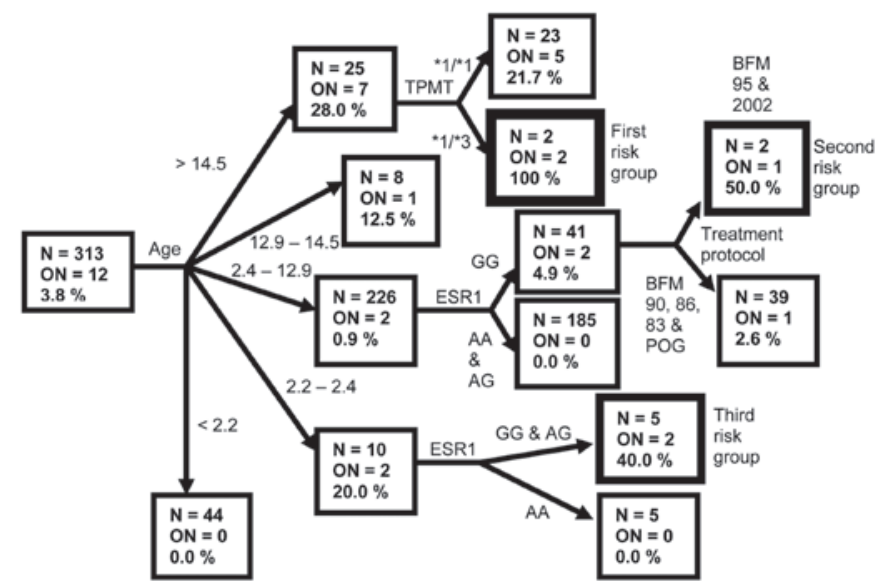

Figure 2. Classification tree for the risk of ON after treatment for childhood acute lymphoblastic leukemia (ALL). Percents represent the incidence of $\mathrm{ON}$ in the given subgroup. Analysis of 313 childhood ALL patients from cancer registry of Slovenia. The classification and regression tree growing method was used for the statistical analysis. N, number of patients in the subgroup; ON, number of osteonecrotic patients in the subgroup; ESR1, estrogen receptor alpha 1; TPMT, thiopurine S-methyltransferase; BFM, Berlin-Frankfurt-Muenster protocol.

ON. Furthermore, in patients $>14.5$ years, a mutated TPMT genotype was associated with increased ON risk, while for children younger than 12.9 years ESRI GG and AG genotypes were the main risk factor for ON.

In the present cohort age was a strong independent risk factor for $\mathrm{ON}$, which is in accordance with numerous studies $(2,4,8,28,29)$. It has been established that adolescents, particularly those $>15$ years, are at highest risk for ALL therapy-induced ON. This may reflect the greater vulnerability of rapidly growing bone in adolescent period (1).

Another independent risk factor in the present study was treatment protocol. More recent treatment protocols (BFM-90, -95 and IC-BFM 2002) had higher incidence of ON compared with older protocols. This could be explained by the differences in drug administration schemes in different protocols. All chemotherapeutic agents that are used in the treatment of ALL were found to reduce the number of osteoblast-like cells in vitro (5), and combinations of different agents had more toxic effects compared with individual agents (30). GCs were found to exhibit the strongest risk of $\mathrm{ON}$ in the clinical setting during ALL therapy (2), although MTX and alkylating agents were also indicated to be associated with $\mathrm{ON}$ risk in certain studies $(2,3,6)$. Therefore, we reviewed all used protocols to detect differences in administration schedule and doses of GC and MTX. The main difference between recent (BFM-90, -95 and IC-BFM 2002) and older (POG, BFM-83 and -86) protocols were higher cumulative doses of GC (particularly dexamethasone) and MTX in recent protocols. This is in accordance with the results of two large childhood cancer survivor studies, in which GC therapy was strong risk factor for ON (2) and patients receiving dexamethasone were more likely to develop ON compared with patients who received prednisone alone $(2,28)$. Another important difference in the IC-BFM 2002 was the introduction of intermittent maintenance therapy (IMT) consisting of weekly MTX $\left(20 \mathrm{mg} / \mathrm{m}^{2}\right)$ and daily 6-MP $\left(50 \mathrm{mg} / \mathrm{m}^{2}\right)$. IMT was administered in all patient risk groups between the blocks of reinduction therapy (25). Introduction of 2 IMT blocks for standard risk and 3 blocks for intermediate risk group into the reinduction therapy resulted in higher cumulative doses of dexamethasone compared to standard reinduction therapy. In accordance with this, the incidence of ON was highest in the IC-BFM 2002 group (15\%), markedly exceeding ON incidence in BFM-90 (8\%) and BFM-95 (5\%) protocols. Notably, all ON patients treated with the IC-BFM 2002 protocol were assigned to the intermediate risk group.

Deactivating polymorphisms in the TPMT gene were a risk factor for ON in patients $>14.5$ years. TPMT is a S-Adenosyl methionine (SAM)-dependent methyltransferse that catalyses the deactivation of thiopurine drugs, such as 6-MP and 6-thioguanine (31). If the TPMT activity is low (such as in the presence of polymorphisms in the TPMT gene), this will result in high concentrations of cytotoxic thioguanine nucleotides (TGN) due to decreased conversion of thiopurine drugs to inactive methylated metabolites (31). The toxic effects of 6-MP on bone and bone cells have been demonstrated in vitro (5) and in vivo (7). 6-MP reduced cell numbers in osteoblast-like cell line MG63 at therapeutically relevant concentrations (5), and in a retrospective study of childhood ALL survivors number of weeks of 6-MP exposure were strongly correlated with hip BMD reduction (7). High concentrations of TGN exert toxic effects on majority of cells and this effect is most pronounced in 


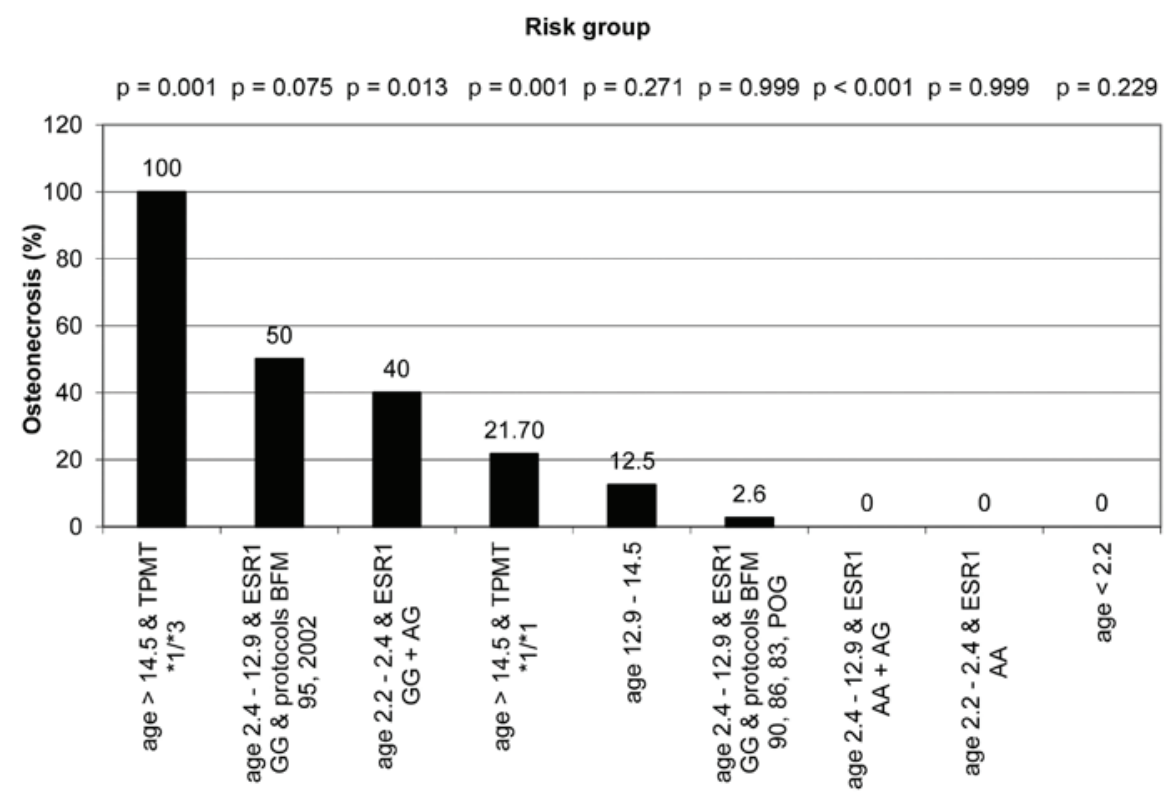

Figure 3. Combined risk factors for osteonecrosis the percentage of patients observed to have osteonecrosis in each risk group (i.e. terminal node of the classification tree); P-values represent comparison to the rest of the observed population. The Fischer's exact test was used for the statistical analysis. TPMT, thiopurine S-methyltransferase; ESR1, estrogen receptor alpha 1; BFM, Berlin-Frankfurt-Münster; POG, pediatric oncology group.

bone marrow cells (32). Since newly formed osteoblasts derive from MSCs residing in bone marrow, we hypothesize that higher levels of TGNs in individuals deficient in TPMT may lead to decreased numbers of MSCs and consequently osteoblasts, leading to the increased $\mathrm{ON}$ risk. In this manner, 6-MP could aggravate the effects of GC on MSCs, which include the reduction of MSC number in bone marrow (33) and increased differentiation of MSCs into adipocytes at the expense of osteogenesis (34). Recently it has also been implicated that MSCs may have an important role in the repair process of osteonecrotic lesion (35). MSCs, cultured in the low-oxygen conditions, resembling those in the osteonecrotic lesion, were found to produce high levels of vasculogenic cytokines (35). Since vascular damage is speculated to be one of the primary ON causes, this induction of the vascularization by MSCs may lead to ON improvement (35). Thus, any additional factors decreasing MSC number or differentiation into osteoblasts may worsen the osteonecrotic process, induced by GC. This could explain why only a small fraction of patients receiving GC therapy develop ON and why a relatively small percentage of patients with positive radiograph and MRI osteonecrotic results are symptomatic (35). In the case of 6-MP therapy of the present study, decreased TPMT activity leads to greater bone marrow toxicity and decreased MSC number, which may impair bone formation and repair the forming osteonecrotic lesion. In addition to increased 6-MP toxicity, the impaired TPMT activity may influence bone toxicity through changes in methylation potential. It has been demonstrated in vitro that decreased activity of SAM-dependent methyltransferases lead to impaired osteoblast differentiation via DNA-methylation independent mechanism (36). Reduced global methylation decreases the activity of transcription factor Runx2, a key regulator of osteoblast differentiation (36).

In the present study, the $X b a I$ polymorphism in the ESRl gene represents an ON risk factor only in pre-pubertal children. In children $<12.9$ years the GG genotype increased ON risk, while AA genotype was protective of ON. ESR1 plays an important role in the regulation of skeletal growth and maintenance of bone mass (37). We investigated a common polymorphism in the ESRl gene $(X b a \mathrm{I})$, which has already been extensively studied $(15,38-40,42)$. A meta-analysis of association studies involving 5,834 subjects showed a correlation of $\mathrm{G}$ allele with higher BMD and decreased risk of fracture (38), while in another large-scale genome-wide study a correlation with fracture that was independent of BMD was observed (15). However, all of the aforementioned studies were performed on adults and not in the context of cancer therapy. In a study by Boot et al, the $X b a$ I AA genotype was associated with lower lumbar spine BMD in healthy children (39). Furthermore, the effect of ESR1 was more pronounced in pre-pubertal children, which is in accordance with the present results. This may be due to the fact that defects in estrogen receptor have more pronounced clinical consequences in the setting of low estrogen concentrations, such as in pre-pubertal children or post-menopausal women (39). However, the present results indicating $X b a \mathrm{I}$ GG genotype as risk factor for ON are not in accordance with the results of a previous study on healthy children where the same genotype was associated with higher lumbar spine BMD. Notably, in the aforementioned study (33), XbaI was associated with lumbar spine BMD, but not with total body BMD. This may be because the spine is rich in trabecular bone, which has a high bone turnover rate, while the body primarily consists of cortical bone with low turnover (39). Notably, patients in the present study had ON of the hip, where the percent of trabecular bone is lower compared with the spine. There is evidence that estrogens have a more marked effect on cortical than on trabecular bone, possibly due to increased expression of ERa in cortical bone (39). The molecular mechanism by which ESRI XbaI 
affects osteoporosis and BMD is unclear; however, there is evidence that it may affect gene transcription. Results of Maruyama et al suggest that the presence of $\mathrm{XbaI} G$ allele can decrease ESR1 activity (40). This is in accordance with the present results that indicate that GG genotype is a risk factor for ON, as decreased levels of estrogen are known to be associated with low BMD and osteoporosis (41). In the context of childhood ALL therapy, polymorphisms in ESR1 were not correlated with BMD, although they were associated with the recovery of lean body mass after the treatment (42). Finally, all of the discussed previous studies examining the effects of ESR1 on BMD were performed on osteoporotic populations. Although osteoporosis and $\mathrm{ON}$ are characterized by low BMD, the pathological mechanism of each diseases is different (1). In osteoporosis, bone resorption and formation are accelerated, while the resorption/formation ratio is increased (43). In ALL therapy-related ON the bone formation is inhibited due to the decreased number of precursor MSCs and their increased differentiation to adipocytes, while at the same time all bone cells are dying at an increased rate due to the decreased level of oxygen and nutrients caused by thrombotic complications (1). This may underlie the discrepancy between the present results regarding osteonecrotic patients and the result of osteoporosis studies in connection to ESR1.

The present exploratory study contained a number of limitations, the first point that needs to be addressed is the number of cases. The study included all pediatric ALL patients from Slovenia from a period of over 30 years. As severe ON is a very rare condition, the number of cases is low despite the lengthy study period. To confirm the present findings, a replication study on another ALL population is required. Secondly, we did not measure BMD in ALL patients as this was not routinely performed during diagnosis and treatment of ALL patients. BMD measurements may offer an additional insight into $\mathrm{ON}$; however, due to the retrospective nature of the study, this was not possible. Thirdly, we employed a hypothesis-based approach and thus did not fully investigate all the possible genetic markers. However, studied genes were selected to cover all three aspects that contribute to the $\mathrm{ON}$ development: Bone metabolism (COL1A1, ESR1, MTHFR), thrombosis (MTHFR, ESRI) and ALL pharmacogenetics (TPMT, MTHFR). Besides these selected genes, there are numerous other genes that may warrant addition study, such as ACP-1, PAI-1 and TYMS; however, due to the limited quantity of the samples these were impossible to perform. From the same reason, we did not fully cover all genetic variations in the studied genes, but included only those that were clinically and epidemiologically most relevant.

In conclusion, the present retrospective exploratory study of 313 childhood ALL patients suggests that greater age and more recent treatment protocols were independent risk factors for ON. Furthermore, different genetic risk factors were identified in specific age groups. While in patients $>14.5$ years TPMT genotype modulated the risk of $\mathrm{ON}$, in children $<12.9$ years ESR 1 genotypes were implicated in the pathogenesis of $\mathrm{ON}$. As these genetic markers have potential prognostic value for the prediction of $\mathrm{ON}$ in pediatric ALL therapy, these findings require confirmation in a replication study with a larger number of $\mathrm{ON}$ patients.

\section{Acknowledgements}

The authors thank Miss Lil Klaas (Erasmus exchange student) for technical assistance and Professor Roger Pain for assistance with English language. The study was funded by the Slovenian Research Agency (grant nos. J3-3615 and L3-2330).

\section{References}

1. Sala A, Mattano LA Jr and Barr RD: Osteonecrosis in children and adolescents with cancer-an adverse effect of systemic therapy. Eur J Cancer 43: 683-689, 2007.

2. Kadan-Lottick NS, Dinu I, Wasilewski-Masker K, Kaste S, Meacham LR, Mahajan A, Stovall M, Yasui Y, Robison LL and Sklar CA: Osteonecrosis in adult survivors of childhood cancer: A report from the childhood cancer survivor study. J Clin Oncol 26: 3038-3045, 2008.

3. Davies JH, Evans BA, Jenney ME and Gregory JW: Skeletal morbidity in childhood acute lymphoblastic leukaemia. Clin Endocrinol (Oxf) 63: 1-9, 2005.

4. Faraci M, Calevo MG, Lanino E, Caruso S, Messina C, Favr C, Iori A, Santaron S, Bonanomi S, Rondelli R, et al: Osteonecrosis after allogeneic stem cell transplantation in childhood. A case-control study in Italy. Haematologica 91: 1096-1099, 2006.

5. Davies JH, Evans BA, Jenney ME and Gregory JW: In vitro effects of chemotherapeutic agents on human osteoblast-like cells. Calcif Tissue Int 70: 408-415, 2002.

6. Tillmann V, Darlington AS, Eiser C, Bishop NJ and Davies HA: Male sex and low physical activity are associated with reduced spine bone mineral density in survivors of childhood acute lymphoblastic leukemia. J Bone Miner Res 17: 1073-1080, 2002.

7. Warner JT, Evans WD, Webb DK, Bell W and Gregory JW: Relative osteopenia after treatment for acute lymphoblastic leukemia. Pediatr Res 45: 544-551, 1999.

8. Relling MV, Yang W, Das S, Cook EH, Rosner GL, Neel M, Howard S, Ribeiro R, Sandlund JT, Pui CH and Kaste SC: Pharmacogenetic risk factors for osteonecrosis of the hip among children with leukemia. J Clin Oncol 22: 3930-3936, 2004.

9. Zalavras CG, Malizos KN, Dokou E and Vartholomatos G: The $677 \mathrm{C}->\mathrm{T}$ mutation of the methylene-tetrahydrofolate reductase gene in the pathogenesis of osteonecrosis of the femoral head. Haematologica 87: 111-112, 2002.

10. French D, Hamilton LH, Mattano LA Jr, Sather HN, Devidas M, Nachman JB and Relling MV; Children's Oncology Group: A PAI-1 (SERPINE1) polymorphism predicts osteonecrosis in children with acute lymphoblastic leukemia: A report from the Children's Oncology Group. Blood 111: 4496-4499, 2008.

11. Mei L, Ontiveros EP, Griffith EA, Thompson JE, Wang ES and Wetzler M: Pharmacogenetics predictive of response and toxicity in acute lymphoblastic leukemia therapy. Blood Rev 29: 243-249, 2015.

12. Hunger SP and Mullighan CG: Acute lymphoblastic leukemia in children. N Engl J Med 373: 1541-1552, 2015.

13. Glueck CJ, Freiberg RA, Fontaine RN, Tracy T and Wang P: Hypofibrinolysis, thrombophilia, osteonecrosis. Clin Orthop Relat Res 19-33, 2001.

14. Ralston SH and de Crombrugghe B: Genetic regulation of bone mass and susceptibility to osteoporosis. Genes Dev 20: 2492-2506, 2006.

15. Ioannidis JP, Ralston SH, Bennett ST, Brandi ML, Grinberg D, Karassa FB, Langdahl B, van Meurs JB, Mosekilde L, Scollen S, et al: Differential genetic effects of ESR1 gene polymorphisms on osteoporosis outcomes. JAMA 292: 2105-2114, 2004.

16. Ralston SH, Uitterlinden AG, Brandi ML, Balcells S, Langdahl BL, Lips P, Lorenc R, Obermayer-Pietsch B, Scollen S, Bustamante M, et al: Large-scale evidence for the effect of the COLIA1 Sp1 polymorphism on osteoporosis outcomes: The GENOMOS study. PLoS Med 3: e90, 2006.

17. Steer CD, Emmett PM, Lewis SJ, Smith GD and Tobias JH: Methylenetetrahydrofolate reductase (MTHFR) C677T polymorphism is associated with spinal BMD in 9-year-old children. J Bone Miner Res 24: 117-124, 2009.

18. Styrkarsdottir U, Halldorsson BV, Gretarsdottir S, Gudbjartsson DF, Walters GB, Ingvarsson T, Jonsdottir T, Saemundsdottir J, Center JR, Nguyen TV, et al: Multiple genetic loci for bone mineral density and fractures. N Engl J Med 358: $2355-2365,2008$. 
19. Abrahamsen B, Jørgensen HL, Nielsen TL, Andersen M Haug E, Schwarz P, Hagen C and Brixen K: MTHFR c.677C >T polymorphism as an independent predictor of peak bone mass in Danish men-results from the odense androgen study. Bone 38 215-219, 2006

20. Maloney KW, Shuster JJ, Murphy S, Pullen J and Camitta BA Long-term results of treatment studies for childhood acute lymphoblastic leukemia: Pediatric oncology group studies from 1986-1994. Leukemia 14: 2276-2285, 2000.

21. Riehm H, Reiter A, Schrappe M, Berthold F, Dopfer R, Gerein V, Ludwig R, Ritter J, Stollmann B and Henze G: The in vivo response on corticosteroid therapy as an additional prognostic factor in childhood acute lymphoblastic leukemia (therapy study ALL-BFM 83). Klin Padiatr 199: 151-160, 1987 (In German).

22. Reiter A, Schrappe M, Ludwig WD, Hiddemann W, Sauter S, Henze G, Zimmermann M, Lampert F, Havers W and Niethammer D: Chemotherapy in 998 unselected childhood acute lymphoblastic leukemia patients. Results and conclusions of the multicenter trial ALL-BFM 86. Blood 84: 3122-3133, 1994.

23. Schrappe M, Reiter A, Ludwig WD, Harbott J, Zimmermann M Hiddemann W, Niemeyer C, Henze G, Feldges A, Zintl F, et al: Improved outcome in childhood acute lymphoblastic leukemia despite reduced use of anthracyclines and cranial radiotherapy: Results of trial ALL-BFM 90. German-Austrian-Swiss ALL-BFM study group. Blood 95: 3310-3322, 2000.

24. Möricke A, Reiter A, Zimmermann M, Gadner H, Stanulla M, Dördelmann M, Löning L, Beier R, Ludwig WD, Ratei R, et al Risk-adjusted therapy of acute lymphoblastic leukemia can decrease treatment burden and improve survival: Treatment results of 2169 unselected pediatric and adolescent patients enrolled in the trial ALL-BFM 95. Blood 111: 4477-4489, 2008.

25. Fronkova E, Mejstrikova E, Avigad S, Chik KW, Castillo L, Manor S, Reznickova L, Valova T, Zdrahalova K, Hrusak O, et al: Minimal residual disease (MRD) analysis in the non-MRD-based ALL IC-BFM 2002 protocol for childhood ALL: Is it possible to avoid MRD testing? Leukemia 22: 989-997, 2008

26. National Cancer Institute Common toxicity criteria manual, version 2.0. Available on-line at: http://ctep.cancer.gov/ protocolDevelopment/electronic_applications/docs/ctcmanual_ v4_10-4-99.pdf Accessed August 3, 2014.

27. Ogino $S$ and Wilson RB: Genotype and haplotype distributions of MTHFR677C $>$ T and 1298A $>$ C single nucleotide polymorphisms: A meta-analysis. J Hum Genet 48: 1-7, 2003.

28. Aricò M, Boccalatte MF, Silvestri D, Barisone E, Messina C, Chiesa R, Santoro N, Tamaro P, Lippi A, Gallisai D, et al: Osteonecrosis: An emerging complication of intensive chemotherapy for childhood acute lymphoblastic leukemia. Haematologica 88: 747-753, 2003.

29. Burger B, Beier R, Zimmermann M, Beck JD, Reiter A and Schrappe M: Osteonecrosis: A treatment related toxicity in childhood acute lymphoblastic leukemia (ALL)-experiences from trial ALL-BFM 95. Pediatr Blood Cancer 44: 220-225, 2005 .
30. Davies JH, Evans BA, Jenney ME and Gregory JW: In vitro effects of combination chemotherapy on osteoblasts: Implications for osteopenia in childhood malignancy. Bone 31: 319-326, 2002.

31. Karas-Kuzelicki N and Mlinaric-Rascan I: Individualization of thiopurine therapy: Thiopurine S-methyltransferase and beyond. Pharmacogenomics 10: 1309-1322, 2009.

32. Maltzman JS and Koretzky GA: Azathioprine: Old drug, new actions. J Clin Invest 111: 1122-1124, 2003.

33. Hernigou P, Beaujean F and Lambotte JC: Decrease in the mesenchymal stem-cell pool in the proximal femur in corticosteroid-induced osteonecrosis. J Bone Joint Surg Br 81: 349-355, 1999.

34. Yin L, Li YB and Wang YS: Dexamethasone-induced adipogenesis in primary marrow stromal cell cultures: Mechanism of steroid-induced osteonecrosis. Chin Med J (Engl) 119: 581-588, 2006.

35. Muller I, Vaegler M, Holzwarth C, Tzaribatchev N, Pfister SM, Schütt B, Reize P, Greil J, Handgretinger R and Rudert M: Secretion of angiogenic proteins by human multipotent mesenchymal stromal cells and their clinical potential in the treatment of avascular osteonecrosis. Leukemia 22: 2054-2061, 2008.

36. Vaes BL, Lute C, van der Woning SP, Piek E, Vermeer J, Blom HJ, Mathers JC, Müller M, de Groot LC and Steegenga WT: Inhibition of methylation decreases osteoblast differentiation via a non-DNA-dependent methylation mechanism. Bone 46: 514-523, 2010.

37. Nakamura T, Imai Y, Matsumoto T, Sato S, Takeuchi K, Igarashi K, Harada Y, Azuma Y, Krust A, Yamamoto Y, et al: Estrogen prevents bone loss via estrogen receptor alpha and induction of Fas ligand in osteoclasts. Cell 130: 811-823, 2007.

38. Ioannidis JP, Stavrou I, Trikalinos TA, Zois C, Brandi ML, Gennari L, Albagha O, Ralston SH and Tsatsoulis A; ER-alpha Genetics Meta-Analysis: Association of polymorphisms of the estrogen receptor alpha gene with bone mineral density and fracture risk in women: A meta-analysis. J Bone Miner Res 17: 2048-2060, 2002.

39. Boot AM, van der Sluis IM, de Muinck Keizer-Schrama SM, van Meurs JB, Krenning EP, Pols HA and Uitterlinden AG: Estrogen receptor alpha gene polymorphisms and bone mineral density in healthy children and young adults. Calcif Tissue Int 74: 495-500, 2004.

40. Maruyama H, Toji H, Harrington CR, Sasaki K, Izumi Y, Ohnuma T, Arai H, Yasuda M, Tanaka C, Emson PC, et al: Lack of an association of estrogen receptor alpha gene polymorphisms and transcriptional activity with Alzheimer disease. Arch Neurol 57: 236-240, 2000.

41. Riggs BL: The mechanisms of estrogen regulation of bone resorption. J Clin Invest 106: 1203-1204, 2000.

42. te Winkel ML, van Beek RD, de Muinck Keizer-Schrama SM, Uitterlinden AG,Hop WC,Pieters R and van den Heuvel-Eibrink MM: Pharmacogenetic risk factors for altered bone mineral density and body composition in pediatric acute lymphoblastic leukemia. Haematologica 95: 752-759, 2010.

43. Raisz LG: Pathogenesis of osteoporosis: Concepts, conflicts, and prospects. J Clin Invest 115: 3318-3325, 2005. 